\title{
El juego como manifestación cuántica: una aproximación a la epistemología infantil*
}

\author{
Desirée López de Maturana Luna**
}

\begin{abstract}
Resumen: La Educación Parvularia en Chile y principalmente su gestión pedagógica, se sitúa en un nuevo escenario que exige de los educadores competencias más humanas que de funcionalidad técnica para adaptarse a las impresiones, la emergencia, la incertidumbre y la complejidad de los procesos epistemológicos infantiles; comprender cómo aprenden los niños, cómo acceden al conocimiento y qué estrategias utilizan para hacerlo, es fundamental para contribuir a un sistema educativo de calidad. Si bien en Chile se han intentado algunas reformas e innovaciones curriculares para la Educación Parvularia con el firme propósito de mejorar la calidad del sistema educativo, aún no está claro, qué es aquello que entorpece el logro de los aprendizajes y pone en peligro la calidad del sistema. En este contexto, resulta interesante centrar la mirada en los juegos infantiles, describir el tipo de interacciones sociales y con el entorno que se producen en estas dimensiones lúdicas e intentar descubrir los indicios epistemológicos que en ellas surjan; descubrir en los espacios de informalidad en que se desarrollan los juegos infantiles algunas aproximaciones que nos contextualicen epistemológicamente, nos permitirá comprender que las prácticas adultistas utilizadas en los espacios educativos formales no impactan significativamente los procesos de aprendizaje de los niños.
\end{abstract}

Palabras clave: Bases Curriculares de la Educación Parvularia, epistemología infantil, interacción lúdica, perspectiva cuántica

\section{The game as quantum manifestation: an approach to children's epistemology}

\begin{abstract}
Nowadays in Chile, initial education, and mainly its pedagogical management, is located in a new scene that demands from educators more human abilities than technical functionality to adjust to impressions, emergencies, uncertainty and the complexity of the epistemological children steps; to understand how children learn, how they get into knowledge and what kind of strategies they use to achieve it, is fundamental to contribute to a quality educational system. Although some curricular reforms and innovations in initial education have been tried in Chile, with the firm purpose to improve the quality of educational system, it is not clear yet what is it that obstructs reaching the learning achievements and jeopardizes the quality of this system. In this scenario, it is interesting to focus on child games, to describe the kind of interaction -social and with the environmentwhich emerges from this recreational dimension, and also try to discover
\end{abstract}

\footnotetext{
* Tesis doctoral en curso. Doctorado en Mediación Pedagógica. ULS. Artículo publicado dentro del contexto del proyecto FONDECYT 1080073: "Complejidades educativas emergentes y caóticas en la escuela lineal”.

** Universidad de La Serena, La Serena, Chile. Email: dlopezdematurana@junji.cl
} 
epistemological signs there; finding at the informal spaces where children games are develop, some approximations that can give us epistemological context, will allow us to know that "adult" practices used on formal educational spaces do not impact significantly in children's learning processes.

Key words: Curriculum Foundations of Preschool Education, children's epistemology, playful interaction, quantum perspective.

\section{O jogo como manifestação cuántica: uma aproximação à epistemología infantil}

Resumo: A Educação Parvularia em Chile e principalmente sua gestão pedagógica, situa-se num novo palco que exige dos educadores concorrências mais humanas que de funcionalidad técnica para se adaptar às impressões, a emergência, a incerteza e a complejidad dos processos epistemológicos infantis; compreender como aprendem os meninos, como acedem ao conhecimento e daí estratégias utilizam para o fazer, é fundamental para contribuir a um sistema educativo de qualidade. Conquanto em Chile tentaram-se algumas reformas e inovações curriculares para a Educação Parvularia com o firme propósito de melhorar a qualidade do sistema educativo, ainda não está claro, que é aquilo que entorpece o lucro das aprendizagens e põe em perigo a qualidade do sistema. Neste contexto, resulta interessante centrar a mirada nos jogos infantis, descrever o tipo de interacções sociais e com o meio que se produzem nestas dimensões lúdicas e tentar descobrir os indícios epistemológicos que nelas surjam; descobrir nos espaços de informalidad em que se desenvolvem os jogos infantis algumas aproximações que nos contextualicen epistemológicamente, permitir-nos-á compreender que as práticas adultistas utilizadas nos espaços educativos formais não impactan significativamente os processos de aprendizagem dos meninos.

Palavras-chave: Bases Curriculares da Educação Parvularia, epistemología infantil, interacção lúdica, perspectiva cuántica

Recibido: 15.01.2010

Aceptado: 07.02.2010

$* * *$

\section{Breve reseña de la reforma curricular de la Educación Parvularia en Chile}

\section{A modo de Marco Referencial}

Las nuevas Bases Curriculares (2000) para la Educación Parvularia, fueron elaboradas por la Unidad de Currículum y Evaluación del Ministerio de Educación a través de la activa participación del sector, donde se elabora una propuesta curricular con aportes de las familias, instituciones normativas y ejecutoras de programas, instituciones formadoras profesionales y técnicas para incorporar en su composición, además de las características y potencialidades de los párvulos, los nuevos elementos sociales, históricos y culturales propios de nuestro país y los nuevos escenarios pedagógicos.

Un aspecto fundamental de esta reforma curricular es la reconstruc- 
ción del concepto de párvulo, que abre espacios a una nueva dialéctica entre la teoría y la práctica pedagógica. Parte por exponer que la infancia es una construcción social críticamente afectada por las ideas, modas y tecnologías con que se cuenta, considerando que dicha construcción conceptual se ha definido, hasta ahora, desde una perspectiva adultista, sin ninguna participación de los niños. Así, la infancia, ha sido definida por relaciones proteccionistas, en las que los adultos son proveedores dominantes y los niños consumidores pasivos ${ }^{1}$. Por el contrario, la visión que surge en este nuevo paradigma es concebir al niño como un co-constructor de conocimientos, de identidad y de cultura, es decir una concepción de niño, como actor social, mucho más integral y potente que requiere de adultos, de instituciones y de pedagogías más potenciadoras; se habla, entonces, del niño como ser humano cuyo organismo es un sistema abierto y modificable, donde la inteligencia no es ya un valor fijo, "sino que constituye un proceso de autorregulación dinámica, sensible a la intervención de un mediador eficiente y el desarrollo del cerebro, depende de un complejo interjuego entre los genes con que se nace, la existencia de un sistema de influencias en ambientes enriquecidos y las experiencias variadas que se tienen" (MINEDUC 2001). La creencia fundamental es que las experiencias tempranas tienen una gran importancia en la arquitectura del cerebro, en su naturaleza, profundización y extensión de las capacidades a lo largo de la vida.

Necesitamos educar y preparar una generación que vivirá en un mundo acelerado, que plantea nuevos requerimientos educativos que están transformando profundamente la sociedad contemporánea, por ejemplo, el impacto causado por las tecnologías de la información, la Internacionalización y el impacto del conocimiento científico. Para dar cuerpo y transformar la variedad de elementos y condiciones sociales y culturales que enmarcan y dan sentido al quehacer educativo a inicios del siglo XXI, han sido elaboradas las Bases Curriculares teniendo como criterio fundante, también, el derecho de la familia de ser la primera educadora de sus hijos"2.

Este marco curricular plantea la formulación de 8 principios pedagógicos cuya orientación vela por dar respuesta y considerar de manera integrada y permanente todos los aspectos biológicos, ontológicos, culturales, tecnológicos y medio ambientales, que conforman los procesos educativos. Estos principios son: Principio de bienestar, Principio de actividad, Principio de singularidad, Principio de potenciación, Principio de relación, Principio de unidad, Principio del significado, Principio de juego.

\footnotetext{
1 “La reforma Educacional de la Educación Parvularia”. Una oportunidad para generar cambios significativos para una mejor calidad. Serie Educación Parvularia 2002: aportes para la reflexión y acción. Ministerio de Educación; División de Educación General. Unidad de Educación Parvularia

${ }^{2}$ Bases Curriculares de la Educación Parvularia. MINEDUC. Unidad de Curriculum y Evaluación. 2001
} 
En relación a los Objetivos generales de la Educación Parvularia, se puede señalar que estos persiguen enunciar explícitamente los grandes desafíos que tenemos hoy para abordar la tarea educativa y pedagógica en los primeros años de vida de las personas. Los verbos utilizados en cada uno de ellos, dan cuenta que la responsabilidad está puesta en los adultos, no obstante implícitamente, se les exige que se reposicionen y contextualicen epistemológicamente con respecto a los niños. Otros componentes o categorías de esta organización curricular son los Ámbitos de experiencias para los aprendizajes, Núcleos de aprendizajes, Objetivos generales y aprendizajes esperados para cada núcleo y definidos en dos Ciclos, desde los tres meses a los tres años y desde los tres años a los seis respectivamente. Estos especifican el "qué se espera que aprendan.” Pueden ser desglosados, especificados o complementados según las características de cada comunidad educativa, las modalidades curriculares que se apliquen y los intereses, necesidades, fortalezas y características de los párvulos; están expresados en términos de definiciones de lo que deben saber o ser capaces de hacer los niños a mediano plazo; permiten diversas formas de manifestación de los aprendizajes acorde a sus posibilidades y singularidades. Las Orientaciones Pedagógicas, procuran fundamentar y exponer criterios para la realización y manejo de las actividades destinadas al logro de los aprendizajes. Además, se incluyen orientaciones para la organización de los contextos de aprendizaje, que son la planificación, la conformación de la comunidad educativa, la organización de los espacios y tiempos educativos y la evaluación.

Este Marco Curricular puede llevar a los educadores a trabajar según sus propias interpretaciones, si bien las definiciones son claras en su intención humanista, igualmente se puede implementar de maneras distintas, por un lado, en congruencia con el sentido educativo de realizar esta tarea confiando en la autonomía ${ }^{3}$ de los niños y por otro, a través del funcionamiento condicionado a un marco curricular rígido, que impone moldes y patrones de vida a los niños, para dirigirlos hacia un camino determinado, hacia un objetivo particular, un aprendizaje esperado, que no es otra cosa, que dejar atrás su originalidad y su condición de niños. La tentación de la aplicación mecánica es la que ha hecho caer en contradicciones vitales a sistemas o propuestas de mejora.

Por el contrario, cuando optamos por seguir pautas humanistas, estamos optando por una educación liberadora, que se preocupa de la plena realización de los niños, de formar su conciencia crítica, de suscitar su inconformidad con lo establecido y de ser ellos mismos, en comunión con los otros y con el mundo.

\footnotetext{
3 José María Alonso define la autonomía como: “...la formación de la conciencia capaz de establecer en si misma el "lugar de control”, de ser el laboratorio donde se elaboran los criterios y los juicios capaces de gobernar a la persona desde su propio dinamismo, sin someterse a los ataques de alienación tan propios de la sociedad consumista y trivializada." (2004:49) José María Alonso (2004) La educación en valores en la institución escolar. Planenación - programación. Universidad de La Salle - Plaza y Valdés, México (2004:49)
} 


\section{La epistemología infantil, elemento fundamental en el currículum de la educación inicial}

Los indicios que nos dan los niños, permiten que nos replanteemos nuestro rol como educadores para que tengamos en cuenta la valoración y el respeto por el trabajo propio del sujeto en situación de aprendizaje (Escudero, 1990 en Soto 1992: 45), la construcción de un currículum formativo en torno a las necesidades y aspiraciones de los sujetos y la creación de un clima de seguridad que les permita interpelarse mutuamente sus respectivas posiciones. Desde este punto de vista, surge como tema fundamental la epistemología infantil, que considera al niño como ser integral e integrado a su entorno natural y social.

El encuentro epistemológico de los niños y de los adultos, no será posible si los educadores no confiamos en que son ellos quienes nos muestran de manera fresca, transparente e ingenua, cómo aprenden y cómo construyen colectivamente el conocimiento; ellos dan espacio a lo emergente, a las propuestas, a la multiplicidad de verdades que surgen de una misma realidad en un armónico caos. Si pudiésemos colorear estas interacciones e interrelaciones, obtendríamos una visión caleidoscópica, como una artística manifestación de la incertidumbre. Lo paradójico, es que esta imagen dinámica, llena de color y emoción, es la que en la escuela se asocia al desorden, al descontrol, a lo feo y anormal. Por ello debe fortalecerse una estructura funcional cuyo objetivo es mantener el status quo, sobre la base de normas y «verdades» incuestionables, que persiguen alcanzar y mantener el poder de tener "todo" bajo control.

Los niños y los adultos estamos construyendo permanentemente conocimientos "cotidianos" 4 independientemente del espacio escolar. Esta actividad constructiva no se detiene con la escolaridad por el sentido y funcionalidad que le otorga la epistemología cotidiana, la que imprime las maneras en que nos aproximamos al conocimiento escolar. Por lo tanto, los niños son personas que asisten a un establecimiento educacional "equipados" con una serie de conceptos y concepciones adquiridas, que le servirán como instrumentos para seleccionar, organizar y establecer relaciones. Ellos construyen el conocimiento y nadie puede sustituirles en esa tarea, del mismo modo que serán ellos, quienes logren traducir a partir de su acervo experiencial la realidad que se les presenta en el contexto de su propia epistemología.

Los niños cuando ingresan a la escuela por primera vez, no sólo

\footnotetext{
${ }^{4}$ María José y Rodrigo José Arnay en su libro La construcción del conocimiento escolar presentan una clara distinción entre el conocimiento cotidiano y el escolar. Señala que ambos tipos de conocimientos, no se distinguen necesariamente por su contenido, sino muy particularmente por su epistemología constructiva, por el tipo de escenario sociocultural en que se construyen y por sus procesos de construcción.
} 
traen un conocimiento “previo”, sino un escenario y una epistemología constructiva "previa” que van a utilizar para construir el conocimiento llamado escolar (Arnay 1997). Razón por la cual, el sentido que los niños le otorgan a los contenidos y a las teorías escolares tendrán una forma propia poco adecuada a los requerimientos de la escuela. Este tipo de epistemología no sigue métodos ni procedimientos científicos de comprobación de hipótesis ni de búsqueda exhaustiva de soluciones, por eso, el conocimiento cotidiano, útil y adaptativo, resulta negativo para su adecuación al medio escolar.

Sin temor a la contradicción, cabe señalar que no obstante lo dicho, la epistemología cotidiana, tiene también su propia rigurosidad; es habitual ver, por ejemplo, que los niños están haciendo sus propias hipótesis, prueban y comprueban; descubren problemas y buscan soluciones, pero siempre en el marco epistemológico que les es propio.

Lo peligroso es construir conocimiento dentro de la escuela tomando en cuenta los conocimientos cotidianos y atribuir significados sólo para convertirlos en una herramienta de anclaje del nuevo conocimiento escolar. Saber qué hacer con este conocimiento en la escuela y la importancia de rescatar el escenario y epistemología correspondiente, es el punto neurálgico que alienta una nueva pedagogía.

Es necesario percibir atentamente los sucesos que acontecen fuera del marco que imponen arbitrariamente los espacios educativos formales, que a la luz de lo señalado, se definen engañosamente como "acuerdos"; poner atención a las conversaciones y a las comunicaciones no verbales que otorgan la riqueza a la experiencia cotidiana y nos permite atravesar "la fachada ilusoria de objetividad" (Harris, 2000:58) para reencontrarnos con esa dimensión subjetiva, humana y sensible que nos hemos acostumbrado a omitir y negar, es urgente, de lo contrario nos alejamos de lo que podríamos definir como aprendizaje real y trascendente.

Esa omisión, es una tendencia peligrosa a la hora de construir y administrar el currículum educacional, porque nos conduce a ordenar la realidad desde lo externo, de forma homogénea, esperable según parámetros previamente establecidos para maximizar la eficacia de los aprendizajes. Evidentemente, este modelo empírico objetivista, garantiza la cuantificación, la medición y las certezas adecuadas para explicar lo que sucede cotidianamente en el mundo y planificar lo que debemos hacer para alcanzar las metas propuestas. Eso sucede, dentro de un contexto no real, sino como una obra de teatro magistral, en que cada uno debe respetar fielmente su guión, donde aquello que resulta verdaderamente significativo, no está, porque surge de la condición epistémica del ser humano, que por no ser medible ni “objetiva”, se invisibiliza. 


\section{Interacciones e interrelaciones lúdicas: aproximaciones a la epistemología infantil}

Cuando los niños están jugando en exposición directa a estímulos que exigen como respuesta funciones mentales superiores, como la abstracción, la representación, las anticipaciones, las combinaciones, las operatorias, etc. están sumergidos y gozando creativamente de las oportunidades que les ofrece la no certeza, la no determinación, lo no establecido; están aprovechando al máximo la incertidumbre y la disfrutan como una instancia de aprendizaje por propia motivación, donde surgen mediadores y líderes que haciendo uso de sus experiencias van generando aprendizajes de manera sinérgica e iterativa, con quienes comparten este espacio relacional.

Desde esta perspectiva, el juego trasciende lo puramente biológico y psicológico, posee un carácter “supra lógico”, que encuentra su sentido más profundo en la cultura, en su función social; es una forma de vida, que se siente, se valora y significa íntimamente. El juego, en tanto instancia de aprendizaje y, por tanto, de socialización, desempeña un papel insustituible en los procesos educativos "informales" que aceptan al "caos" como factor esencial e imprescindible.

Lamentablemente, el juego utilizado en la escuela, fluye reglamentado externamente y no a través del imprevisible fluir de las interacciones infantiles. No se trata de negar la importancia que el niño acepte normas externas, sino de privarlo del gozo de la búsqueda casual, ocasional y fortuita. La no aceptación del carácter casual del juego, responde a un modelo dicotómico de pensar la realidad, reflejo del paradigma de la ciencia moderna donde se materializa la "polarización categorial" de: subjetivo-objetivo; interior-exterior; espíritu-naturaleza; autonomía-heteronomía. Esta dificultad histórica que se desprende del método moderno es obviamente notable en el campo de las disciplinas humanas y, entre ellas, afecta de modo especial al objeto de la educación, a sus métodos, como a la acción del educador (Vitar 1990:186).

La epistemología cotidiana o la educación informal, así denominada desde la perspectiva estructurante y formalizadora de la escuela, ha existido desde siempre como ingrediente fundamental de la vida del ser humano quién establece relaciones e interacciones sociales que se autoorganizan sincrónicamente. En este terreno incierto los sujetos fluyen naturalmente, enseñan y aprenden de forma holística, exploran creativamente en lo incierto y encuentran el saber en la ignorancia.

La física cuántica entrega un criterio epistemológico que niega a las teorías científicas la facultad de generar algo parecido a una ley natural. Si lográsemos este cambio de óptica, nos permitiría relacionarnos permanentemente y actuar en los espacios educativos formales, de manera más libre y menos sometida a la imposición determinista de presagiar cierta regularidad en el comportamiento futuro de los hechos, considerando que los he- 
chos del mundo no responderían a ningún tipo de condicionamiento que hiciera conjeturar alguna estructura en su composición o desarrollo, cuestión que es tan aplicable a los hechos de la naturaleza como a los sociohistóricos (Popper 1994 en Ortega 2007)

Desafortunadamente nuestra condición de adultos se expresa viviendo el devenir del tiempo y el espacio, buscando las mejores formas y nuevas estrategias para "hacerle frente" a la vida, mientras los niños, transitan y fluyen dinámicamente en ese mismo espacio y tiempo, en una dimensión histórica - experiencial, constructiva y placentera.

\section{Una experiencia de aproximación epistemológica}

El juego libre de los niños, cuando surge de ellos, sin la influencia de los adultos, se organiza en base a formas innatas de acciones, movimientos y percepciones, que provienen de la historia evolutiva de la especie humana $\mathrm{y}^{5}$ siendo estas formas de dinámica corporal una herencia conductual ancestral, las configuraciones de movimientos surgen de nuevo, cada vez, en cada niño o niña asociadas a su vivir humano, a su conciencia de sí, de la conciencia social y de la conciencia de mundo.

Un ejemplo de lo señalado es la siguiente experiencia:

En vísperas de Fiestas Patrias, una Educadora a cargo de un nivel Sala Cuna ${ }^{6}$; tenía la tarea de preparar una actividad con los niños de 1 a 2 años para el acto correspondiente, se propuso que en conjunto con ellos demostrarían sus inmensas capacidades.

Los observó atentamente y descubrió su dinámica diaria. Sus configuraciones de movimientos eran rítmicas y circulares, fueran estas, colectivas o individuales. Con eso, se dio a la tarea de buscar una música que se ajustara a ese ritmo y a ese movimiento; encontró una canción tradicional del sur de Chile, llamada "El Pavo"” . De ahí en adelante el trabajo sólo consistió, en ponerles la música y aportarles pañuelos de colores para enfatizar el ritmo y el movimiento. La construc-

\footnotetext{
${ }^{5}$ Maturana, H. y Verden Zoller, G. (1993) Amor y Juego. Fundamentos olvidados de lo humano. Desde el patriarcado a la democracia, Capítulo V. El Desarrollo. El juego libre del niño y la filogenia

${ }^{6}$ El nivel de sala cuna es el primer nivel de la educación Parvularia y se subdivide en sala cuna menor, donde se educan lactantes de 3 meses a 1 año y sala cuna mayor para lactantes de 1 a 2 años.

${ }^{7}$ El estribillo de la canción dice: "el pavo con la pava, se picotean, se picotean y la pava no permite que le hagan rueda, que le hagan rueda...”, esta melodía y el ritmo que la acompaña, provoca la configuración del movimiento circular.
} 
ción de la coreografía fue maravillosa. No hubo necesidad de diseñar, ni estructurar el espacio, ni acordar la finalización, puesto que desde esa edad, los niños ya son capaces de captar e intuir las notas tónicas ${ }^{8}$.

Aquí, los niños nos demuestran que cuando hablamos de generar instancias de aprendizaje desde los primeros años de vida, no nos estamos refiriendo a exigirles tareas para las cuales no están preparados, sino de ofrecerles experiencias oportunas que favorezcan el desarrollo de sus propias capacidades.

La experiencia narrada, puede ser una actividad más, que pasa inadvertida, o ser considerada como una oportunidad para conocer y comprender cómo los niños van manifestando su conducta inteligente, sin complicaciones, ni frustraciones. El análisis puede desarrollarse desde muy diversa perspectivas, contextos, paradigmas o teorías. Gardner ${ }^{9}$, por ejemplo, plantea la connotación general que se la ha atribuido a la inteligencia, circunscribiéndola fundamentalmente, al predominio de lo lingüístico y de la lógica matemática; por ello propone una nueva noción de inteligencia a partir de siete áreas en que se encontraría el potencial intelectual, éstas, sin excepción, aparecen en la propuesta de éstos niños y estas niñas cuyas edades no superaban los 2 años.

Si bien la teoría de Gardner ha sido cuestionada ${ }^{10}$, es igualmente, una poderosa contribución a la pedagogía, porque hace evidente y explícito que se debe diversificar las formas a través de las cuáles los niños puedan acceder al conocimiento. En el ejemplo descrito, se observa claramente que el ritmo musical, surge del ritmo universal y por lo mismo se da en todo proceso vital. En este caso los niños disfrutaron del movimiento como manifestación de su ley biológica y espiritual; por ello su relación con la música fue natural, instintiva y positiva.

\footnotetext{
${ }^{8}$ Existe una nota tónica en la cual, si no siempre comienza la melodía, en ella de ordinario termina y descansa

${ }^{9}$ Gardner, H. La mente no escolarizada. Paidós, Barcelona. Las inteligencias a las que alude son: verbal/lingüística, vinculada a la expresión hablada o escrita; musical/rítmica, basada en el reconocimiento de patrones tonales, sonidos y la sensibilidad para reproducir y crear ritmos; lógico/matemática, relacionada con el pensamiento inductivo y deductivo, los números y el reconocimiento de patrones abstractos; viso/espacial, centrada en el sentido de la visión. Tiene la capacidad de crear representaciones e imágenes mentales; corporal/ kinestésica, vinculada al movimiento físico y al conocimiento del cuerpo; interpersonal, centrada en la relación persona a persona y a la comunicación; intrapersonal, vinculada a los estados internos, la autorreflexión, la metacognición y la conciencia de situarse en el tiempo y en el espacio (1997: 45).

${ }^{10}$ Se la cuestiona por estar centrada sólo en demostrar la estrecha relación entre ubicaciones encefálicas y el procesamiento de la información o el conocimiento, y por ubicar en zonas cerebrales específicas cada una de las inteligencias. Ref. Texto "La reforma Curricular de la educación Parvularia” Pontificia Universidad Católica de Chile. Teleduc 2002
} 
Esta construcción infantil nos permite descubrir que los niños experimentaron, vivieron la música por medio de una interpretación corporal de ella; construyeron una propuesta plástica al estímulo musical que resultó de una relación directa entre mente, espíritu y cuerpo, en forma totalmente empírica, esta experiencia, muestra como los niños viven la "relajación” en vez de "tensión"; el "ritmo interior" en vez de “compás”; la "creación” en vez de "imitación”, donde el cuerpo pasa a ser un intermediario entre el sonido, la emoción y el pensamiento. Dalcroze ${ }^{11}$ (en Volosky, L. 1980: 230)

Es necesario tener presente que aunque pudiésemos creer que efectivamente estamos utilizando el juego como estrategia de aprendizaje, reconociendo toda su vertiente biológica cuyas definiciones conceptuales pueden ser del todo claras y ciertas, no sea una actividad opresiva y mecánica. Desde el punto de vista de la epistemología infantil, el juego es con sentido propio, genuino y espiritual, que por su condición inmaterial, es complejo e indefinible; existe porque "cancela la determinabilidad absoluta" que lo hace pensable y comprensible"; es aquel, que manifiesta su dimensión estética cuyo orden radica en el movimiento, en la figura, en lo bello, en el ritmo y en la armonía (Huizinga 1972: 13 - 14)

En esta crítica al inductivismo, se entiende que si bien es posible identificar elementos previos que posibiliten la capacidad de predecir, en estricto rigor epistemológico, la capacidad de predicción es imposible de llevarse a efecto, toda vez que el mundo es irrepetible y cada hecho o proceso guarda una existencia única. La tesis indeterminista, supone que "el concepto de libertad, es homologable y equivalente a la imposibilidad de determinar el curso futuro de los hechos; y segundo, que la creación humana, o en su efecto, la capacidad del ingenio creador del artista o el científico, no pueden existir en condiciones sociopolíticas que coercionen dicha facultad” (Popper Op.cit. Ortega 2007)

\section{Conclusiones}

La unidad y la diversidad son dos perspectivas inseparables fundantes de la educación, por lo tanto, hablar de educar es también hablar de una educación que se proyecta hacia un tiempo y un espacio futuro, desconocido e insospechado. Es mirar hacia una realidad incierta que nos aguarda inevitablemente, que la podemos abordar con temor y recelo, buscando la manera de conservar el control, o por el contrario, conquistarla, transformándonos en participantes creativos para vivir en plenitud, abriéndonos a dimensiones creativas y sinérgicamente armoniosas. De esa manera, la fí-

${ }^{11}$ Pedagogo y compositor Suizo, que vivió entre los años 1865 y 1950. Se formó en el Conservatorio de Ginebra. Creador del Método Dalcroze (1907 - 1914), cuyo concepto pedagógico, propone una nueva forma de aprender más, desarrollando el sentido plástico y musical de acuerdo con la naturaleza de los niños y la música. 
sica, la química, la matemática, la geometría y todas aquellas ciencias llamadas exactas, no son sólo un conocimiento propio de una elite, sino, parte de la vida misma que se ha ido construyendo a través de la relación del ser humano con su entorno.

En este contexto, considerar el análisis fenomenológico permite comprender la trampa en que caemos los seres humanos a medida que nos vamos haciendo adultos, nos civilizamos y nos "culturizamos" desarrollando con maestría "ciertos estados de conciencia”, pre-suponiendo y suponiendo respecto de objetos, situaciones, acontecimientos y comportamiento de los otros.

El desafío es alcanzar la "suspensión fenomenológica” y prescindir de los supuestos para ver la realidad con un "'ojo inconsciente", que es lo que vuelve a los niños 'fenomenólogos” más naturales que los adultos (Husserl en Ruiz, D. 1997). La interpretación de los estados de conciencia pudiese parecer contradictoria, en tanto los estados de conciencia de los niños, son distintos de la conciencia adulta. Mientras los niños están absortos en una tarea disfrutando en plenitud, los adultos estamos empeñados en calzar en un estado de conciencia exógeno, de apariencia racional, "normal”; punto de disonancia y de desencuentro epistemológico, que incide indiscutiblemente en la calidad de los procesos educativos. 


\section{Bibliografía}

José María Alonso (2004), La educación en valores en la institución escolar. Planenación - programación. Universidad de La Salle - Plaza y Valdés, México.

Amay, J. y Rodrigo M. J. (1997), La construcción del conocimiento escolar Paidós, Barcelona.

Calvo, C. (2008), Del mapa escolar al territorio educativo. Disoñando la escuela desde la educación. Nueva Mirada Ediciones. $3^{\mathrm{a}}$ edición, La Serena.

Delval, J. (2000), Aprender en la vida y en la escuela, Morata, Madrid.

Gardner, H. (1997), La mente no escolarizada, Paidós, Barcelona.

Idem (1995), Inteligencias Múltiples, Paidós, Barcelona.

Huizinga, J. (1972), Homo Ludens, Ed. Alianza, Madrid.

Maturana, H; Verden Zoller, G. (1993), Amor y Juego. Fundamentos olvidados de lo humano. Desde el patriarcado a la democracia, Editorial Instituto de Terapia Cognitiva, Santiago.

Ortega, C. (2007), "Sobre la incompatibilidad indeterminismo-libertad en el racionalismo crítico de Karl Popper" en Revista de Ciencias Sociales, $\mathrm{N}^{\circ} 18$, Universidad Arturo Prat, Iquique.

Pontificia Universidad Católica de Chile (2002), La reforma Curricular de la educación Parvularia. Teleduc. Volosky, L; Rosenthal, I. y Mira, P (1980), Manual para la Educación de Párvulos, Ed. Universitaria, Santiago.

Wolff, M. (1994), La cotidianeidad, Ediciones Cátedra, Madrid.

Zohar, D. (1996), El yo cuántico. Naturaleza humana y conciencia definida por la fisica moderna, Publimex, Ciudad de México. 